\title{
Joint multiband signal detection and cyclic spectrum estimation from compressive samples
}

\author{
Lebing Pan ${ }^{1,2^{*}}$, Shiliang Xiao ${ }^{1,2}$, Xiaobing Yuan ${ }^{1}$ and Baoqing $\mathrm{Li}^{1}$
}

\begin{abstract}
This paper focuses on wide-sense stationary signal processing within a compressive sensing framework, proposing a new method of compressive sampling fast Fourier transform (FFT) accumulation method (CS-FAM). Depending on how it is applied, CS-FAM has one or two steps, allowing for versatility in multiband signal detection and parameter extraction. In the first step, the active sub-bands are detected using multiple measurement vectors (MMVs) and multiuser detection is achieved using a bandwidth constraint. In applications where it is required, such as in estimations of carrier frequency, symbol rate, or modulation format identification, the second step can be used to reconstruct the cyclic spectrums of each user individually. Based on the results of first step, parameter extraction is performed by searching for peaks in the cyclic spectrum rather than by the usual method of setting a threshold. Compared to other cyclic feature detection methods based on sub-Nyquist sampling, CS-FAM is low in complexity, allowing for practical implementation. Based on the results of the first step, parameter extraction from the cyclic spectrum is performed by searching for peaks rather than by setting a threshold. Although CS-FAM can only be employed for multiband signal detection, compared to other cyclic feature detection methods based on sub-Nyquist sampling, it is low in complexity, which makes practical implementation possible. Numerical simulations are presented to demonstrate the robustness of CS-FAM's multiband signal detection and the effectiveness of its cyclic spectrum estimation against both sampling rate reduction and noise uncertainty.
\end{abstract}

Keywords: Multiband signal detection; Parameter extraction; Cyclic spectrum estimation; Compressive sampling

\section{Introduction}

Signal detection and parameter estimation of wideband radio signals are increasingly important aspects of communication systems such as electronic countermeasures [1], carrier tracking [2], and spectrum sensing [3]. However, conventional detection techniques using standard analogto-digital converters (ADCs) have been primarily designed to capture narrowband signals [4]. Wideband sensing based on Nyquist sampling is similarly unsuitable in such applications, often yielding either an insupportable sampling burden or an unacceptable sampling complexity. Additionally, because of this, wideband receivers require a high sampling rate, which typically results in high power consumption. In contrast, analog front-ends have often been used to capture sparse signals in scenarios such as cognitive radio networks

\footnotetext{
* Correspondence: panlb@mail.sim.ac.cn

${ }^{1}$ Key Laboratory of Wireless Sensor Networks, Shanghai Institute of Microsystem and Information Technology, Chinese Academy of Sciences, 865 Changning Road, Shanghai 200050, China

${ }^{2}$ University of Chinese Academy of Sciences, No.19A Yuquan Road, Beijing 100049, China
}

\section{Springer}

(c) 2014 Pan et al.; licensee Springer. This is an Open Access article distributed under the terms of the Creative Commons Attribution License (http://creativecommons.org/licenses/by/4.0), which permits unrestricted use, distribution, and reproduction in any medium, provided the original work is properly credited. sampling (also known as compressive sensing [5]), conducted using low rate ADCs, has been extensively employed to detect wideband signals. With the underlying assumption of signal sparsity, this technology is capable of complete signal recovery from a small number of linear measurements [6].

A number of methods for radio signal detection using compressive sampling have been presented in the literature. Several studies have focused on use of blind multiband signal reconstruction [7] and power spectrum sensing [8-11]. These works aim to detect active subbands distributed over a wide frequency spectrum, which is a crucial step in dynamic spectrum access $[12,13]$. Other literature has proposed methods for cyclic spectrum estimation using sub-Nyquist sampling. Also known as cyclic feature detection, these methods pertain not only to spectrum sensing but also to symbol rate estimation, carrier frequency estimation, and modulation identification [14-17]. In [18], the spectrum correlation function (SCF) 
(also known as cyclic spectrum.) of each narrowband signal was analyzed individually. This scheme recovered signals in the time domain using compressive sampling and then computed the SCF at the Nyquist rate. The linear relationship between the SCF and the time-varying covariance function was first derived in [19] and [20]. This operation makes the sparse approximation algorithms generated by compressive sensing available for cyclic feature detection. The scheme in [21] used the symmetry property of the cyclic autocorrelation function for blind spectrum sensing. On another side, the time smoothed algorithm known as the fast Fourier transform (FFT) accumulation method (FAM) was presented in [22] for effective cyclic spectrum estimation based on the Nyquist sampling. A practical implementation of this algorithm for spectrum sensing, presented in [23], showcased its high efficiency and low complexity. In this paper, a two-step compressive sampling FAM (CSFAM) scheme that employs multiple measurement vectors (MMVs) is presented. Multiband detection is accomplished in the first step by means of a modified version of the periodogram method using sub-Nyquist samples, while cyclic spectrum estimation is performed in the second step.

The main characteristics of CS-FAM are as follows: (i) based on the FAM framework, this scheme has low complexity, which is important because cyclic spectrum analysis can lead to high complexity due to the use of two-dimensional spectrum correlation computations, especially from compressive samples. For example, the methods in [20] required matrices proportional to $N^{4}$. However, in implementations such as high-resolution cyclic spectrum estimation by the order of $N \times N$, the memory requirements are quite large, making cyclic spectrum analysis especially difficult. (ii) Conventional cyclic spectrum estimation methods require front-end components capable of high rate sampling. In contrast, in this work, the detection problem is addressed by using a low rate sampling device to capture sub-Nyquist samples. Another advantage of CS-FAM is that performing multiuser detection in the first step eliminates the effect of signal superposition in the multiuser cyclic spectrum, whereas conventional methods require an additional signal separation process. (iii) Due to the use of power spectrum estimation, the multiband signal detection process is robust, compared to other methods based on signal recovery from compressive sampling, which are sensitive typically to noise. For example, the power spectrum blind sampling (PSBS) method used in [9] and [10] demonstrated poor estimation performance in inactive bands, which also led to high complexity in matrix operations. (iv) The process of CS-FAM is flexible in regards to practical application. Multiuser detection is achieved in only one step and, based on the results of the first step, parameter estimation is accomplished in the second step by searching for the peaks in the cyclic spectrum rather than by setting a threshold.

The rest of the paper is organized as follows: Section 2 provides the system model along with the FAM algorithm and compressive sampling framework. CS- FAM is then developed within the framework of FAM. Signal detection is addressed in Section 3. A simple signal recovery algorithm, simultaneous orthogonal matching pursuit (SOMP) is employed for multiband detection. A vector indicating the power distribution of the analog input is then computed from the iteration of SOMP. Following this, active sub-bands are identified using a constant false-alarm detector, and multiuser detection is achieved using a bandwidth constraint. If the signal is reconstructed, the second step of CS-FAM, cyclic feature detection, can be performed. Simulations are presented in Section 4 to demonstrate the detection performance of this scheme, followed by a summary in Section 5.

\section{System model}

Modulated signals have a built-in periodicity, which is characterized as cyclostationary [24]. The auto-correlation function $R_{x}(t, \tau)=E\{x(t) * x(t+\tau)\}$ (* is the complex conjugate operator), which is periodic in the time domain with a period $T_{0}$, is given by

$$
R_{x}\left(t+T_{0}, \tau\right)=R_{x}(t, \tau) .
$$

The cyclic auto-correlation function (CAF) is represented in terms of Fourier coefficients as

$$
R_{x}^{\alpha}(\tau)=\frac{1}{T_{0}} \int_{-T_{0} / 2}^{T_{0} / 2} R_{x}(t, \tau) e^{-j 2 \pi \alpha t} d t
$$

where $\alpha \triangleq\left\{\mathrm{k} / \mathrm{T}_{0}\right\}_{k \in Z}$ represents the cyclic frequencies. The SCF is defined by

$$
S_{x}^{\alpha}(f)=\int_{-\infty}^{\infty} R_{x}^{\alpha}(\tau) e^{-i 2 \pi f \tau} d \tau .
$$

Wide-sense stationary signals can be detected from stationary interference because general stationary signals do not possess cyclic stationary characteristics. The advantage of cyclostationary feature detection lies in its ability to separate the signal of interest (SOI) from noise and interference in the spectral correlation plane, making it well suited for modulation recognition and parameter estimation.

The framework of CS-FAM is illustrated in Figure 1. Because the detection method in this paper is based on a process of cyclic spectrum estimation using compressive 


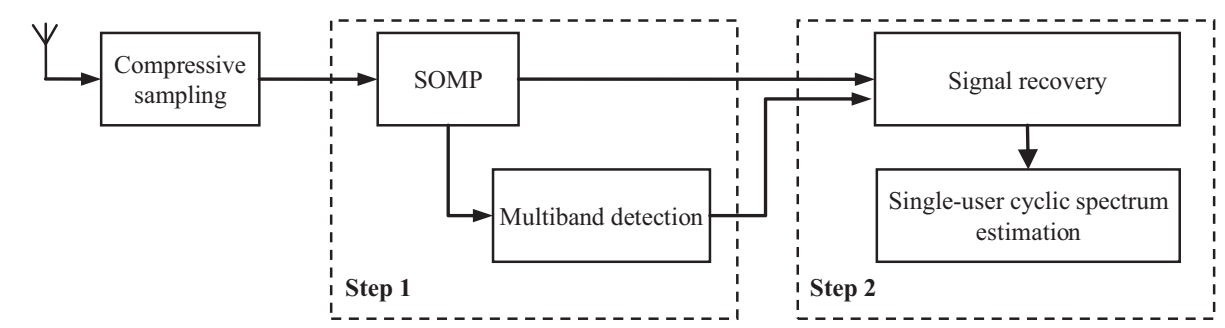

Figure 1 Flexible signal detection by CS-FAM.

sampling, we will introduce the FAM algorithm and compressive sampling framework first.

\subsection{The FAM algorithm}

Compared to other methods used to estimate SCF, time smoothed algorithm is computationally efficient for general cyclic spectral analysis [22]. Based on the time smoothed cyclic periodogram, the parameter estimation is calculated with a data tapering window of the length $T$ sliding over the data for a time span of $\Delta t$ and $\Delta t=N^{\prime} T_{s}$ ( $N^{\prime}$ is the length of $x[n])$, where $T_{s}$ is the sample interval. The SCF of the digital signal $x[n]$ is defined as $S_{x}^{\alpha}(f)=\sum_{b=-\infty}^{\infty} R_{x}^{\alpha}(b) e^{-j 2 \pi f b}$ with the discrete Fourier transform (DFT). The cyclic periodogram [25] is defined as

$$
S_{x}^{\alpha}(n, f)=X_{T}\left(n, f_{1}\right) X_{T}^{*}\left(n, f_{2}\right)
$$

where $f=\left(f_{1}+f_{2}\right) / 2$ and $\alpha=f_{2}-f_{1} . X_{T}(n, f)$ denotes a complex demodulator, which is expressed as

$$
X_{T}(n, f)=\sum_{r=-N^{\prime} / 2}^{N^{\prime} / 2-1} w(r) x(n-r) e^{-i 2 \pi f(n-r) / N^{\prime}},
$$

where $w(n)$ is a data tapering window, which is well accomplished by using a Hamming window. The limited cyclic spectrum with the discrete-time span of $N^{\prime}$ is defined in terms of the time smoothed cyclic periodogram as

$$
S_{x}^{\alpha}(n, f)_{N^{\prime}}=\sum_{r=n-N^{\prime} / 2}^{n+N^{\prime} / 2-1} X_{T}\left(r, f_{1}\right) X_{T}^{*}\left(r, f_{2}\right) g(n-r)
$$

where $g(n)$ is a data tapering window of length $N^{\prime}$. An implementation based on time smoothed cyclic periodogram is illustrated in Figure 2. As shown in [25] and [26], if the time windows $w(n)$ and $g(n)$ are properly normalized, the time smoothed cyclic periodogram converges to the cyclic spectrum in the limit as follows:

$$
\lim _{\Delta f \rightarrow 0} \lim _{N^{\prime} \rightarrow \infty} S_{x}^{\alpha}(n, f)_{N^{\prime}}=S_{x}^{\alpha}(f)
$$

In order to obtain a substantial reduction in random effects using a time-smoothed approach, the time-spectrum resolution product must significantly exceed unity

$$
\Delta t \Delta f>>1
$$

Thus, substantial observation time is needed to obtain a reliable SCF estimate, where a smaller spectral resolution is required to resolve the individual features of the SCF. The output filter $g(n)$ is not as crucial as the input filter $w(n)$. Therefore, for simplicity, $g(n)$ is taken to be a rectangular window, as is commonly done in FAM algorithms. The computational efficiency of (6) can be improved by decimating the outputs of the filter $w(n)$. With a decimating factor $D,(6)$ is modified to

$$
S_{x}^{\alpha}(D n, f)_{N^{\prime}}=\sum_{r=-L / 2}^{L / 2-1} X_{T}\left(r D, f_{1}\right) X_{T}^{*}\left(r D, f_{2}\right)
$$

$L=N^{\prime} / D$. The filter $w(r)$ ensures that no aliasing takes place after $D$-decimation [27-29]. Since the outputs of channelization are oversampled by a factor of $N$, the sampling rate can be reduced to $f_{s} / D, D \leq N$ before aliasing occurs $[22,28]$. The effects of these parameters on the FAM algorithm are analyzed in [22], which discusses resolution, reliability, and computation reduction. In this paper, we have selected $D=N$ to satisfy the compressive sampling framework expressed by (12). However, the filter is always not ideal. Then, the leakage will influence the estimation performance. While the selection $D=N$ leads to a slight increase in cycle leakage, it significantly reduces complexity. Considering the frequency shifting of the production sequence from $\alpha$ to $\alpha+q \Delta \alpha$, where $q$ is an integer, (9) becomes

$$
S_{x}^{\alpha+q \Delta \alpha}\left(n, f_{i}\right)_{N^{\prime}}=\sum_{r=-L / 2}^{L / 2-1} X_{T}\left(r, f_{l}\right) X_{T}^{*}\left(r, f_{k}\right) e^{-i 2 \pi r q / L}
$$

Here, $f_{l}$ and $f_{k}$ are the frequency values in correlating two spectral components. The FAM estimate computed in (10) corresponds to $\alpha=\left(f_{l}-f_{k}\right)$ and $f_{j}=\left(f_{l}+f_{k}\right) / 2$. The length of $w(n)$ is $N$ and the frequency resolution is $\Delta f=$ $f_{s} / N$, where $f_{s}$ is the sampling frequency. The other 


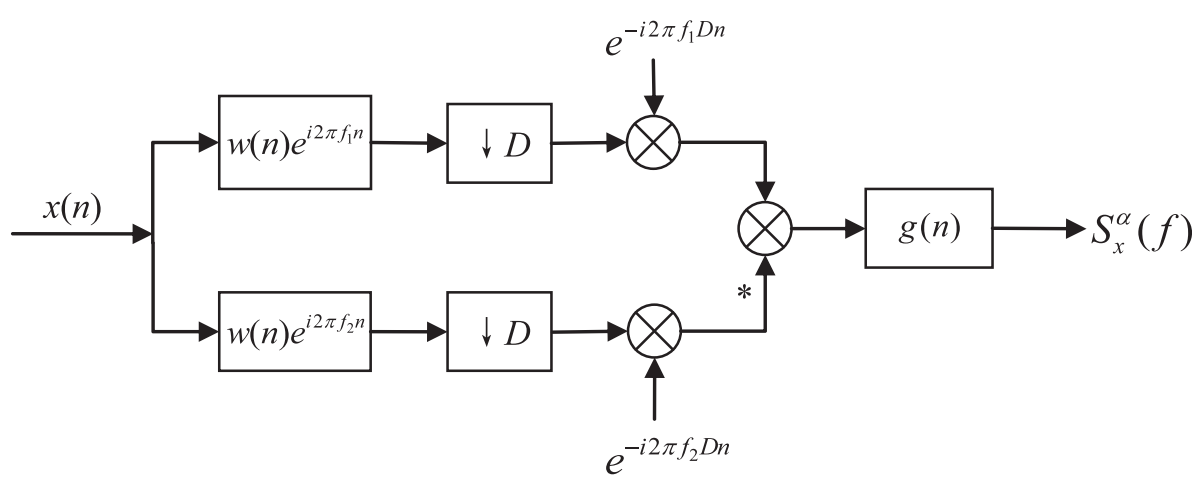

Figure 2 Implementation of time smoothed cyclic periodogram.

dimension $\alpha$ describes the cyclic frequency and the resolution $\Delta \alpha=f_{s} / N^{\prime}$. The FAM is a computationally efficient algorithm by adopting $N$-point FFT operation to (5) after decimating and $L$-point FFT to (10), respectively.

Figure 3a presents a block diagram of the FAM algorithm. The first step includes channelization using data tapering and the $N$-point FFT operation. Data channelization divides the input $x[n]$ into $L$ segments where the size of each segment is $N$. The complex demodulator of (5) after decimating is computed by the $N$-point FFT operation in the first step of Figure 3, which is derived as

$$
\begin{aligned}
\mathbf{X}[f]_{N \times L}=\mathbf{F W} & \mathbf{X}_{0}[n] \\
& =\mathbf{F W}\left(\mathbf{F}^{-1} \mathbf{F}\right) \mathbf{X}_{0}[n] \\
& =\mathbf{W} \mathbf{X}_{0}[f],
\end{aligned}
$$

where $n$ and $f$ denotes the data is expressed in the time domain and in the frequency domain, respectively. $\mathbf{F}$ is the $N$-points of the DFT matrix and $\mathbf{F}_{(i, j)}=\left[e^{-j 2 \pi i j / N}\right] . \mathbf{X}_{0}$ $[f]=\mathbf{F X}_{0}[n] \cdot \overline{\mathbf{W}}=\mathbf{F} \mathbf{W} \mathbf{F}^{-1}$ and $\mathbf{F}^{-1}$ is the $N$-points of the IDFT matrix. $\mathbf{W}$ is a diagonal matrix whose diagonal elements are determined from the $N$-point Hamming window. $\mathbf{X}_{0}[n]$ is the data matrix of size $N \times L$, which is generated from $x[n]$ using channelization. $x[n]$ is the digital signal from the Nyquist sampling.

There are three operations in the second step of FAM as shown in Figures 2 and 3. The complex demodulators are downshifted in frequency to baseband by down conversion. The SCD function is then estimated by multiplying its complex conjugate. The second FFT is a smoothing operation, which is executed by $L$-point FFT. More details on this process are given in [22,24-26]. In this paper, the proposed method is based on the framework of FAM from subNyquist samples as presented in Figure $3 \mathrm{~b}$. The process of obtaining $\mathbf{X}_{0}[f]$ from compressive samples in the first step of CS-FAM is discussed in following section, while the merits of CS-FAM's second step are presented in Section 3.2.

\subsection{Compressive sampling}

According to compressive sampling theory, a small amount of signal data obtained through inner-product operations can be used to reconstruct the original signal with overwhelming accuracy [5,6]. The holy grail of compressed sampling is an acquisition device that exploits signal structure in order to reduce the sampling

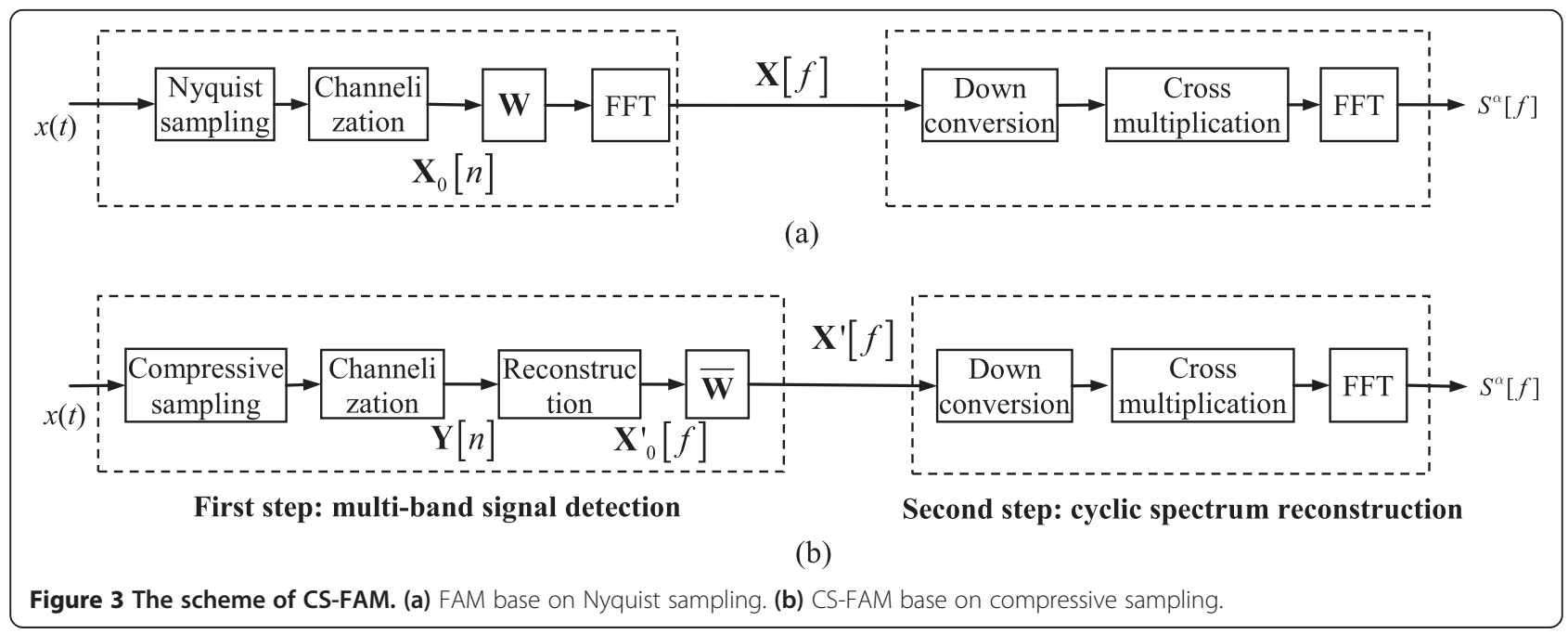




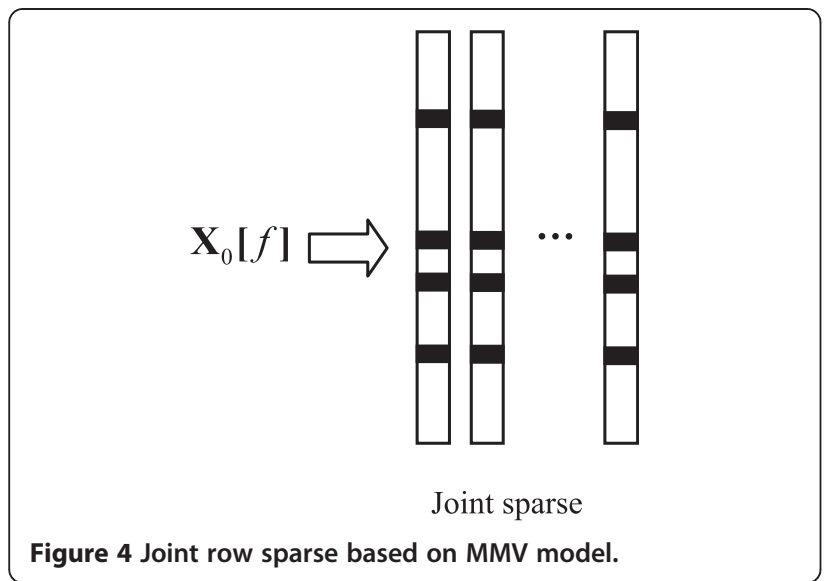

rate. An example of this is the analog-to-information converter (AIC) [30], which is used in place of the ADC in the front-end of a sampling device. As illustrated in Figure $3 \mathrm{~b}$, using an AIC, the output from compressive sampling is expressed as

$$
\mathbf{Y}[n]=\mathbf{A X}_{0}[n],
$$

where $\mathbf{A}$ is a measurement matrix of the size $M \times N$ $(M<N)$, the elements of which are randomly selected. A measurement matrix obeying the restricted isometry property (RIP) in [6] can be used for CS-FAM (e.g., Gaussian random matrix or \pm 1 distribution matrix obeys the RIP with high probability). In this paper, the elements are selected from $\{-1,1\}$, which facilitates hardware implementation by using integrate-and-dump processes [31]. The average sampling rate is equal to the Nyquist rate multiplied by $M / N$. F is invertible. Therefore, we can have

$$
\begin{aligned}
\mathbf{Y}[n] & =\mathbf{A F}^{-1} \mathbf{F X}_{0}[n] \\
& =\mathbf{\Phi} \mathbf{X}_{0}[f],
\end{aligned}
$$

where $\boldsymbol{\Phi}=\mathbf{A F}^{-1}$. The output of the first step in CSFAM is derived by $\mathbf{X}^{\prime}[f]=\overline{\mathbf{W}} \mathbf{X}_{0}{ }^{\prime}[f]$ from (11) as illustrated in Figure $3 \mathrm{~b} . \mathbf{X}_{0}{ }^{\prime}[f]$ is the sparse approximate matrix of $\mathbf{X}_{0}[f]$, which is recovered from compressive samples $\mathbf{Y}[n]$ and only contains the signal in active bands. $\mathbf{X}_{0}{ }^{\prime}[f]$ is computed in Section 3.2.

3 Joint signal processing from compressive samples In this article, multiband detection and parameter extraction are performed in the frequency domain. The process of signal reconstruction using the MMV model will be introduced first. Due to the sparsity of wideband signals, only a few rows of $\mathbf{X}_{0}[f]$ contain large values, denoted as signals with additive noise. In the other rows, where only noise is present, the values are very small. In this study, we let $K$ be the number of rows where the signal is present in $\mathbf{X}_{0}[f]$, and the value of $K$ is small because there are only a few active sub-bands in the whole frequency range. This has given rise to the problem of sparse signal recovery using the MMV model [31] to analyze joint row sparsity, as shown in Figure 4 and which will be addressed below. However, it has been shown that signal recovery rate is greatly increased by using the MMV rather than the single measurement vector (SMV) model. The results in [32-34] reveal the advantages of the MMV model, including the key assumption that the support (i.e., the indexes of the signal entries) is identical in every column of $\mathbf{X}_{0}[f]$. The literature also endorses increasing $L$ for better recovery performance.

Many effective algorithms have been proposed for solving the MMV problem, such as convex relaxation methods using mixed norms, greedy search mechanisms, and sparse Bayesian learning. Compared to other methods, the algorithms based on greedy search mechanisms have the advantageous characteristic of low complexity. Therefore, we selected the simple algorithm SOMP [35], which has proven that under certain conditions, the OMP algorithm can find the sparsest representations from the MMV model with computational efficiency. The SOMP algorithm is modified in this paper as follows:

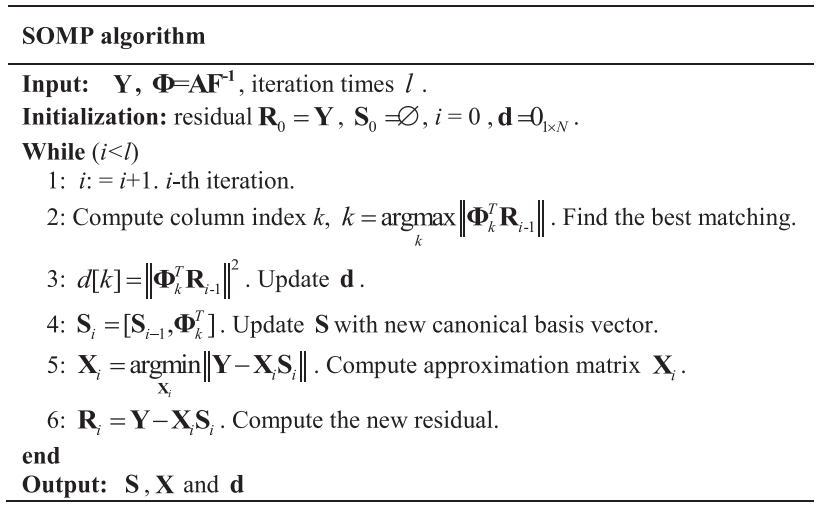

where (.) ${ }^{T}$ denotes the matrix (or vector) transpose and $\boldsymbol{\Phi}_{k}$ is the $k$-th row of $\boldsymbol{\Phi} . \mathbf{X}$ is used to obtain the sparse approximate matrix $\mathbf{X}_{0}{ }^{\prime}[f]$ in Section 3.2. The iteration times $l$ should be larger than $K$. If $K$ is unknown, $l$ should be chosen such that it is sufficiently large. To distinguish between signal and noise measurements, in this paper, the threshold $\lambda_{k}$ is computed by (23). Therefore, in practice, we can give the iteration a termination condition of $d[k]<\lambda_{k}$. When the best matching column 
in $\boldsymbol{\Phi}$ is found in step 2 of SOMP, $\left\|\boldsymbol{\Phi}_{k}^{T} \mathbf{R}_{i-1}\right\|$ indicates the value of $\left\|\mathbf{X}_{k}\right\|$, where $\left\|\mathbf{X}_{k}\right\|$ is the $k$-th row in $\mathbf{X}$. Therefore, it is possible to use $\mathbf{d}$ to identify the active sub-bands using a technique that is similar to the periodogram method, as shown in Figure 5b.

\subsection{Multiband signal detection from compressive samples} If $M$ is large enough $(M>2 K$ is an empirical condition for SOMP), $\mathbf{X}$ is accurately recovered by SOMP with high signal-to-noise ratio (SNR). Constant false-alarm rate energy detection is employed for multiband detection using the periodogram method [36]. The decision statistic for energy detection in the $k$-th row of $\mathbf{X}$ is

$$
\tilde{d}[k]=\frac{1}{L} \sum_{l=0}^{L-1}\left|X_{(k, l)}\right|^{2} \quad(k=1,2, \ldots, N),
$$

where $X_{(k, l)}$ is the $(k, l)$-th element in $\mathbf{X}$. In the following, the detection model is represented by

$$
\begin{array}{llr}
\mathrm{H}_{0}: & \tilde{d}_{0}=n_{0} & \text { signal absent } \\
\mathrm{H}_{1}: & \tilde{d}_{1}=s+n_{0} & \text { signal present }
\end{array}
$$

where $s$ is signal sample, and $n_{0}$ is circular complex zeromean Gaussian independent and identically distributed (i.i.d.) noise with a variance of $\sigma^{2}$. With this model, $\tilde{d}_{0}$ is the sum of the squares of $L$ independent identically distributed zero-mean Gaussian random variables. Therefore, the model produces a random variable using a chisquared distribution that has $L$ degrees of freedom. To achieve predefined sensing performance, the sample frame number $L$ is always large. Therefore, we can use the central limit theorem to approximate $\tilde{d}_{0}$ as the Gaussian function

$$
\tilde{d}_{0} \sim \mathcal{C N}\left(\sigma^{2}, \frac{2 \sigma^{4}}{L}\right)
$$

A binary hypothesis test is then formulated using a decision rule, resulting in

$$
\begin{array}{ll}
\mathrm{H}_{0}: & \tilde{d}[k]<\lambda \\
\mathrm{H}_{1}: & \tilde{d}[k] \geq \lambda .
\end{array}
$$

The threshold $\lambda$ is chosen according to a target falsealarm probability $P_{F A}$ where $P_{F A}=P_{r}\left\{\tilde{d}>\lambda \mid \mathrm{H}_{0}\right\}$ and is given by

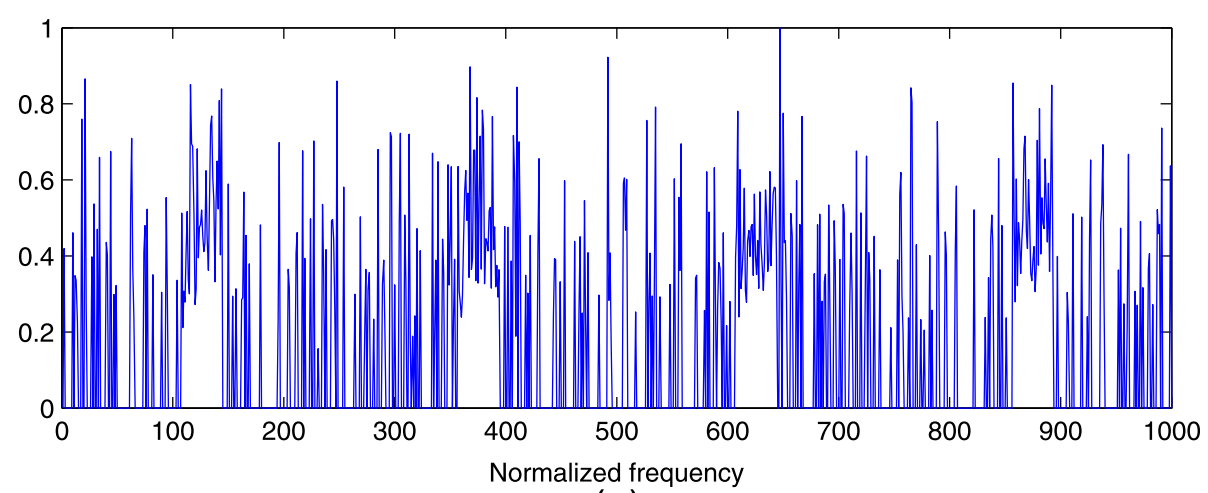

(a)

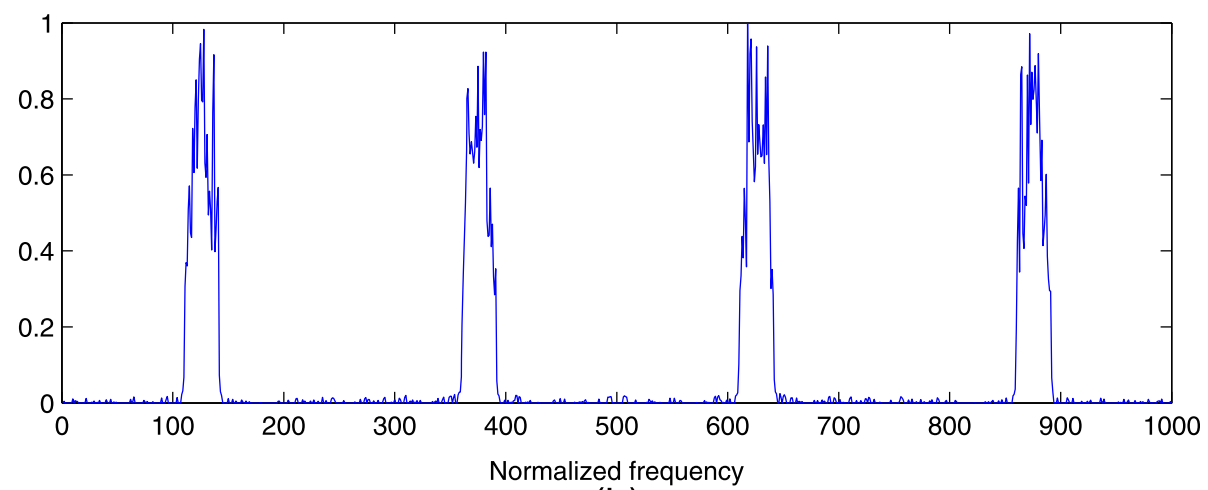

(b)

Figure 5 The decision statistic by SOMP algorithm. (a) The results computed by (14). (b) The results computed by the step.3 of SOMP. 


$$
\lambda=\left(1+\sqrt{\frac{2}{L}}\right) \sigma^{2} Q^{-1}\left(P_{F A}\right) .
$$

where $Q($.$) stands for the tail probability of the Gaussian$ distribution

$$
Q(x)=\int_{x}^{\infty} \frac{1}{2 \pi} \exp \left(-\frac{u^{2}}{2}\right) d u
$$

However, it must be noted that the recovery performance of compressive sensing is sensitive to the number of sampling branches and to the SNR [37]. When $M<$ $2 K$ or the noise level is large, $\mathbf{X}$ cannot be accurately recovered by SOMP and errors may be introduced into $\tilde{d}$, as illustrated in Figure 5a. Performance is even worse if the $\mathrm{SNR}<0 \mathrm{~dB}$. Therefore, in our scheme, we employ the vector $\mathbf{d}$ from SOMP as the input to ensure testing accuracy. The approximate value of the residual $\mathbf{R}_{i}$ is obtained using steps 4 to 6 of SOMP.

$$
\mathbf{R}_{i}=\mathbf{B X},
$$

B is a submatrix of $\Phi$ by removing the columns same with $\mathbf{S}$ (S is derived from SOMP algorithm). Then,

$$
d[k]=\left\|\boldsymbol{\Phi}_{k}^{T} \mathbf{B X}\right\|^{2}
$$

where $\boldsymbol{\Phi}_{k}$ is the $k$-th column of $\boldsymbol{\Phi}$. The correlation between two different columns of the sampling matrix is weak, i.e., $\boldsymbol{\Phi}_{k}^{T} \boldsymbol{\Phi}_{k} \gg \boldsymbol{\Phi}_{k}^{T} \boldsymbol{\Phi}_{k^{\prime}}\left(k^{\prime} \neq k\right)$, which satisfies the basic RIP condition for compressive sampling [6]. The element in $\mathbf{A}$ is selected from $\{1,-1\}$, so the sign of the elements in $\boldsymbol{\Phi}$ is usually random and $\boldsymbol{\Phi}_{k}^{T} \boldsymbol{\Phi}_{k^{\prime}}$ is close to zero. We then have

$$
\begin{array}{r}
d[k] \approx \sum_{l=0}^{L-1}\left\|\boldsymbol{\Phi}_{k}^{T} \boldsymbol{\Phi}_{k} X_{(k, l)}\right\|^{2} \\
=\rho_{k} \sum_{l=0}^{L-1}\left\|X_{(k, l)}\right\|^{2},
\end{array}
$$

where $X_{(k, l)}$ is the $(k, l)$-th element of $\mathbf{X}, \rho_{k}=$ $\left\|\boldsymbol{\Phi}_{k}^{T} \boldsymbol{\Phi}_{k}\right\|^{2}$. The threshold of decision statistic $d[k]$ is

$$
\lambda_{k}=\left(1+\sqrt{\frac{2}{L}}\right) \rho_{k} \sigma^{2} Q^{-1}\left(P_{F A}\right) .
$$

Taking this into account, the binary hypothesis test (16) is rewritten as

$$
\begin{array}{ll}
\mathrm{H}_{0}: & d[k]<\lambda_{k} \\
\mathrm{H}_{1}: & d[k] \geq \lambda_{k} .
\end{array}
$$

In a multiuser network, a single user signal usually occupies successive frequency bands in high-resolution spectrums. Therefore, $\mathbf{d}$ can be divided into $N_{g}$ groups where the length of $i$-th group is $b_{i}$, the number of users is $N_{g}$, and the $i$-th user occupies bandwidth $B_{i}$. To eliminate false spurs in the decision results with the threshold (23), a multiband signal bandwidth constraint is given by

$$
\begin{aligned}
& B_{i}=\frac{b_{i}}{N} f_{s} \geq \frac{b_{m w}}{N} f_{s} \\
& \Delta B \geq \frac{b_{m i}}{N} f_{s},
\end{aligned}
$$

where $b_{m w}$ is minimum frequency bin and $b_{m i}$ is minimum interval frequency bin. $\frac{b_{m w}}{N} f_{s}$ is minimum bandwidth of a user and $\frac{b_{m i}}{N} f_{s}$ is minimum bandwidth interval between two adjacent users.

\subsection{Cyclic spectrum estimation}

Another objective of the SOMP algorithm is to reconstruct $\mathbf{X}_{0}[f]$, which involves signal recovery from compressive samples in the frequency domain. However, using CS-FAM for signal detection differs from using the SOMP algorithm. SOMP directly assigns $\mathbf{X}_{0}[f]=\mathbf{X}$, where $\mathbf{X}$ is the output of SOMP. In contrast, according to the first step of CS-FAM, the row index of $\mathbf{X}_{0}[f]$ indicating only the presence of signal is known. The sparse approximate matrix $\mathbf{X}_{0}{ }^{\prime}[f]$ is computed by

$$
\begin{array}{r}
\mathbf{X}_{0}^{\prime}[f]=\operatorname{argmin}_{\mathbf{X}}\left\|\mathbf{Y}-\mathbf{X} \boldsymbol{\Phi}^{\prime}\right\| \\
\text { s.t. } \quad \mathbf{X}_{k}=\mathbf{0}_{1 \times L}, \quad k \in \mathbf{V} .
\end{array}
$$

$\mathbf{X}_{k}$ is the $k$-th row of $\mathbf{X}$, the elements of which are set to zero, as it indicates only the presence of noise. $\boldsymbol{\Phi}^{\prime}$ is the submatrix of $\boldsymbol{\Phi}$ with removing the columns whose index $k$ supports $k \in \mathbf{V}$. The element of set $\mathbf{V}$ are defined by (24) as the row index of $k$, s.t. $d[k]<\lambda_{k}$. Equation (26) is then solved using the least square (LS) method assuming $M>K$. It is obvious that if the iteration of SOMP algorithm is ended by the termination condition of $d[k]<\lambda_{k}$, we can have $\mathbf{X}_{0}{ }^{\prime}[f]=\mathbf{X}$, where $\mathbf{X}$ is the output of SOMP.

\section{Table 1 Operation complexity of the second step}

\begin{tabular}{lcc}
\hline & FAM & CS-FAM \\
\hline Down conversion & $2 L N$ & $2 K L$ \\
Cross multiplication & $L N^{2}$ & $L \sum_{i=1}^{N_{g}} b_{i}^{2}$ \\
FFT & $N^{2} \frac{L}{2} \log (L)$ & $\frac{L}{2} \log (L) \sum_{i=1}^{N_{g}} b_{i}^{2}$ \\
\hline
\end{tabular}


Table 2 Peak pairs of digital modulation

\begin{tabular}{lc}
\hline Modulation & Peak pairs $(\boldsymbol{f}, \boldsymbol{a})$ \\
\hline BPSK & $\left(f_{c}, \frac{1}{T}\right),\left(0,2 f_{c}\right),\left(0,2 f_{c} \pm \frac{1}{T}\right)$ \\
MSK & $\left(f_{c}, \frac{1}{T}\right),\left(0,2 f_{c} \pm \frac{1}{T}\right)$ \\
AM & $\left(0,2 f_{c}\right)$ \\
QAM & $\left(f_{c}, \frac{1}{T}\right)$ \\
\hline
\end{tabular}

If the approximate matrix $\mathbf{X}_{0}{ }^{\prime}[f]$ is obtained, cyclic spectrum estimation is processed in the second step. Cyclic feature detection in CS-FAM aims to extract parameters rather than to merely detect whether a signal is present or not.

The complexity of the second step is greatly reduced because only $K$ rows are nonzero following (26). Compared to FAM, which is used to estimate the whole span of the spectrum, CS-FAM computes solely the occupied frequency regions of each user. The operation complexity of cyclic spectrum estimation in the second step is illustrated in Table 1. Due to $\sum_{i=1}^{N_{g}} b_{i}=K \ll N$, this complexity of the second step is substantially reduced.

Different modulation formats result in different type of spectral peaks. The pairs of the peaks exhibited by for some common digital modulations [38] are summarized in Table 2, where $f_{c}$ is carrier frequency and $1 / T_{b}$ is symbol rate. If the occupied frequency areas have been identified in the first step, the peaks can be detected in the second by searching within the support range rather than by setting a threshold with the allowable falsealarm rate. For example, if a user with band of $\left(f_{c}-f_{a}, f_{c}\right.$

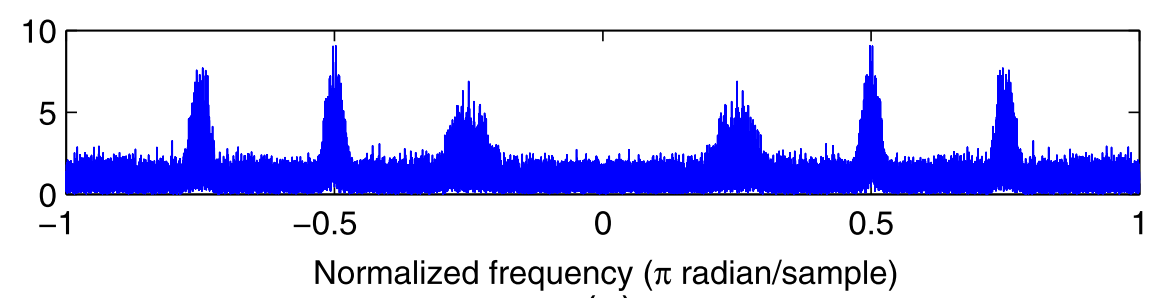

(a)

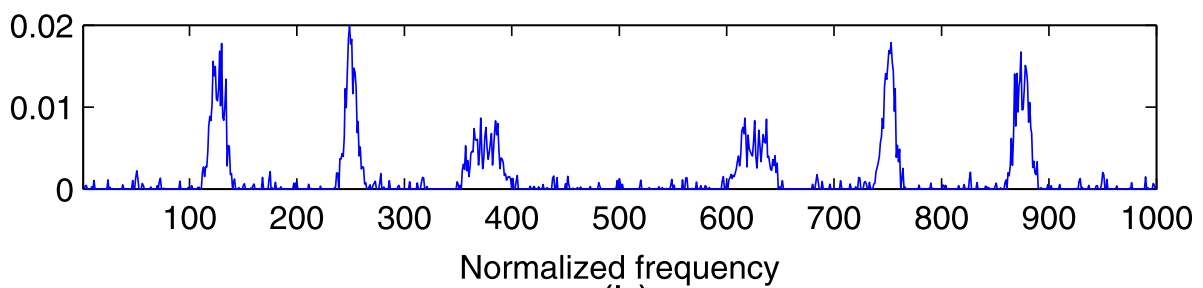

(b)

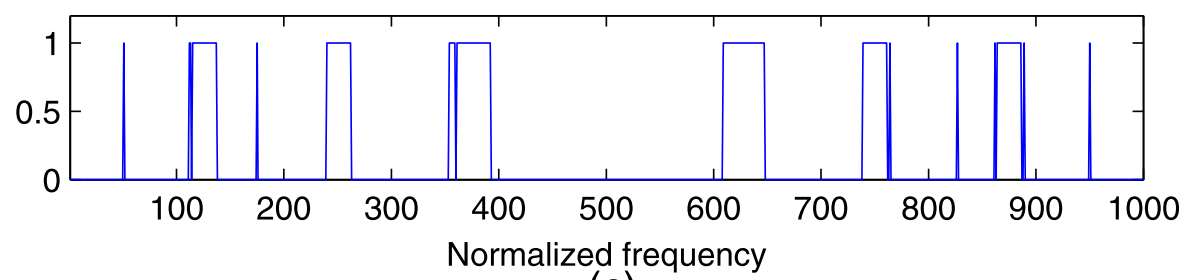

(c)

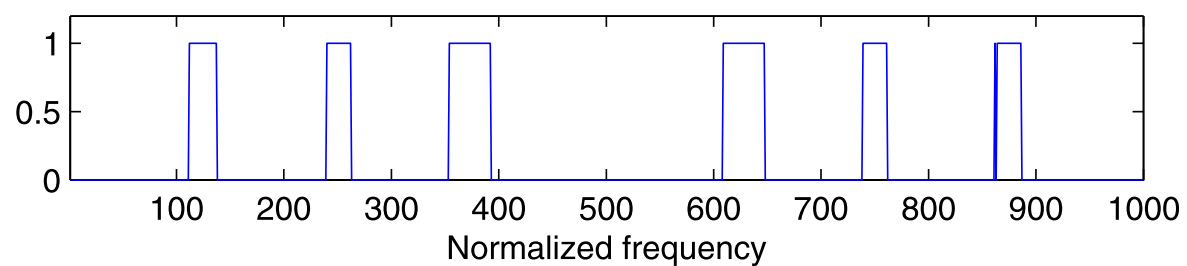

(d)

Figure 6 The process of spectrum sensing in the first step of CS-FAM. (a) The spectrum of original multiband signal, $S N R=0 \mathrm{~dB}$. (b) The results of $\mathbf{d}$ computed by SOMP algorithm. (c) The decision results with the constant false-alarm detector. (d) Eliminate the false spurs by bandwidth constraint. 
$\left.+f_{a}\right)$ is detected from the first step of CS-FAM, the support range of its cyclic spectrum [24] is given by

$$
S_{z}^{\alpha}(f)= \begin{cases}0 & || f|-| \alpha / 2 \| \leq f_{c}-f_{a} \quad \text { or } \\
S_{x}^{\alpha}(f) & \begin{array}{l}
|| f|+| \alpha / 2|| \leq f_{c}+f_{a} \\
\text { otherwise }
\end{array}\end{cases}
$$

Once the peaks are obtained, modulation classification is performed. Some literature on the subject of automatic modulation classification based on cyclic spectrum analysis is summarized in [39]. The symbol rate and carrier frequency are obtained after the modulation format is identified. Because CS-FAM separates multi-signals in the first step, the multiuser cyclic spectrum reconstruction problem of conventional methods becomes simply multiple operations of a single user problem, avoiding signal separation from the whole cyclic spectrum. Performing multiuser detection in the first step of CS-FAM also avoids cyclic spectrum superposition of different users, which may lead to false peaks.

\section{Detection performance and simulation results}

In this section, we demonstrate the performance capacity of the proposed method with performance test results. In Section 4.1, we examine multiband signal detection performance from compressive samples, assuming that the received signals contain only zero-mean Gaussian i.i.d. noise. Section 4.2 is dedicated to examining cyclic spectrum estimation performance.

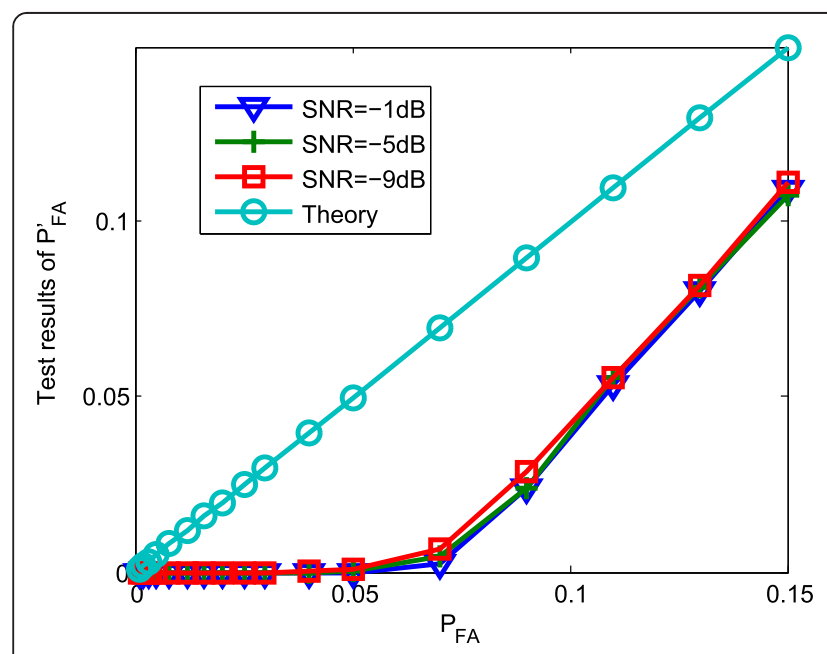

(a)

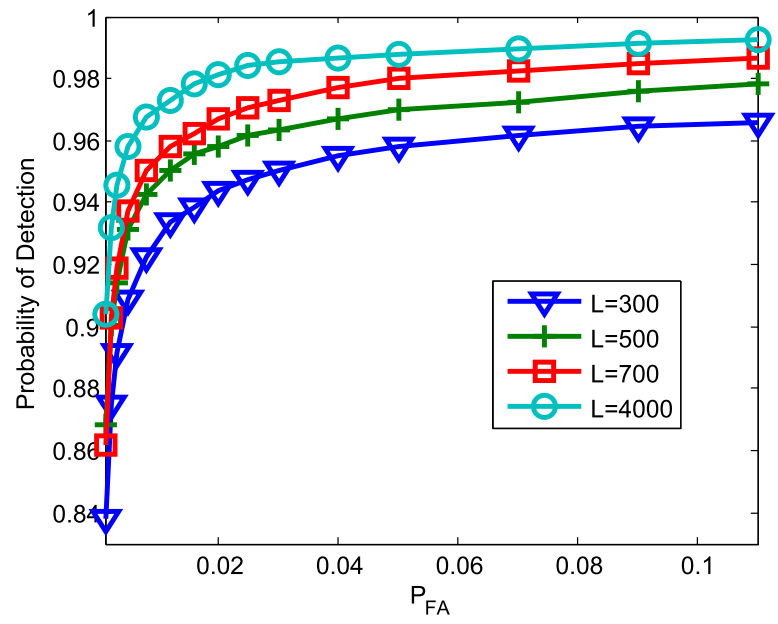

(c)

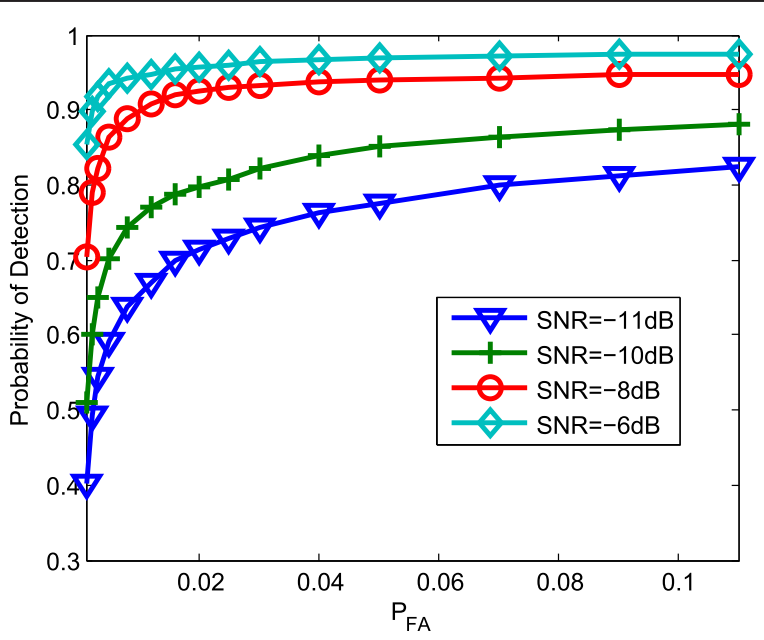

(b)

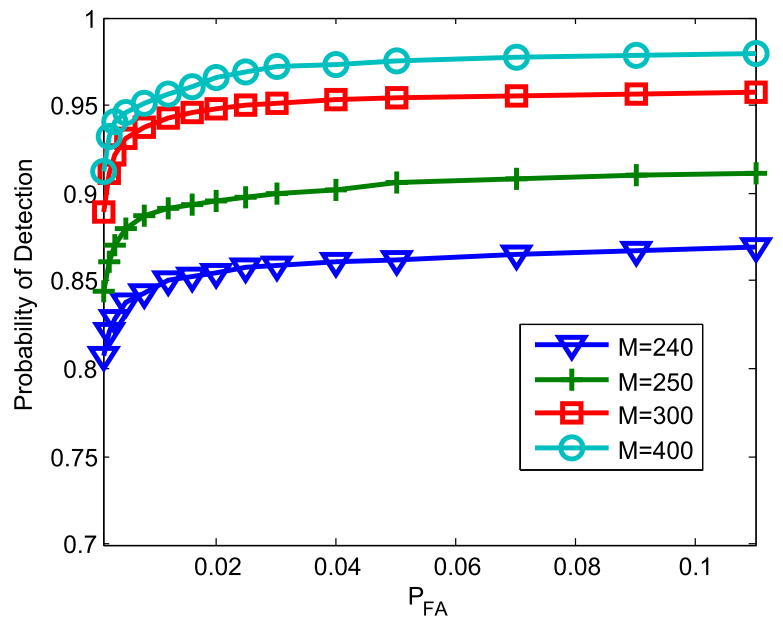

(d)

Figure 7 The spectrum sensing performance of CS-FAM. (a) The test results of probability of false-alarm for various SNR. (b) The detection performance for various SNR. (c) The detection performance for various sensing time. (d) The detection performance for various number of sampling branches. 


\subsection{Multiband signal detection performance}

The process of multiband detection using CS-FAM is illustrated in Figure 6. The analog input content was provided by three binary phase shift keying (BPSK) modulated signals, $N=1,000, M=400$, and $L=50$, shaped by a raised-cosine filter. Figure 6 a shows the spectrum of the received signals, which were transmitted through the additive Gaussian white noise (AWGN) channel. The SNR was $0 \mathrm{~dB}$. The normalized frequency in Figure $6 \mathrm{~b}, \mathrm{c}, \mathrm{d}$ is presented with the resolution of $f_{s} / N$, where $f_{s}$ is the sampling frequency. The result of $\mathbf{d}$, computed by the SOMP algorithm, is presented in Figure 6b. We then applied the constant false-alarm detector with $P_{\mathrm{FA}}=0.05$. The signal detection results are shown in Figure $6 c$, where it can be seen that the false-alarm detector identified a number of false spurs. Figure $6 \mathrm{~d}$ presents the final detection results following elimination of false spurs using a bandwidth constraint of $b_{m w}=b_{m i}=2$. All of the active sub-bands were detected and all three users identified.

An evaluation of the performance of the CS-FAM test is shown in Figure 7. The reference line of Theory in Figure 7a was set by $P^{\prime}{ }_{\mathrm{FA}}=P_{\mathrm{FA}}$, so it presents as a diagonal line. Because using the bandwidth constraint eliminated some of the false spurs, the probability of false-alarm occurrence was lower in the test than in the theoretical value computed by (23). In addition, the rate of falsealarm occurrence using CS-FAM was identical under various noise levels.

Detection performance of the test is presented in Figure 7c, d. The multiband signal contained two BPSK modulated signals. The main lobe of each signal occupied the spectrum from $-0.032 \pi$ to $0.032 \pi$ in the baseband. The threshold was set by (23) according to the target false-alarm rate. Figure $7 \mathrm{~b}$ shows the detection performance under different noise levels, illustrating that the detector demonstrates better performance in higher SNR scenarios. The quality of detection is improved by increasing sensing time, as shown in Figure 7c. However, the results also show that this effect is limited when $L$ is very large. Detection performance with various compression ratios is shown in Figure $7 d$, which indicates that the ratios are improved by increasing the number of sampling branches.

As mentioned before, the CS-FAM multiband detection method is based on power spectrum estimation. Therefore, a comparison between CS-FAM and PSBS $[9,10]$ is presented in Figure 8. As illustrated in Figure 8a, the probability of false alarms in the test was higher when using PSBS than when using CS-FAM, meaning that the PSBS method has poor estimation performance

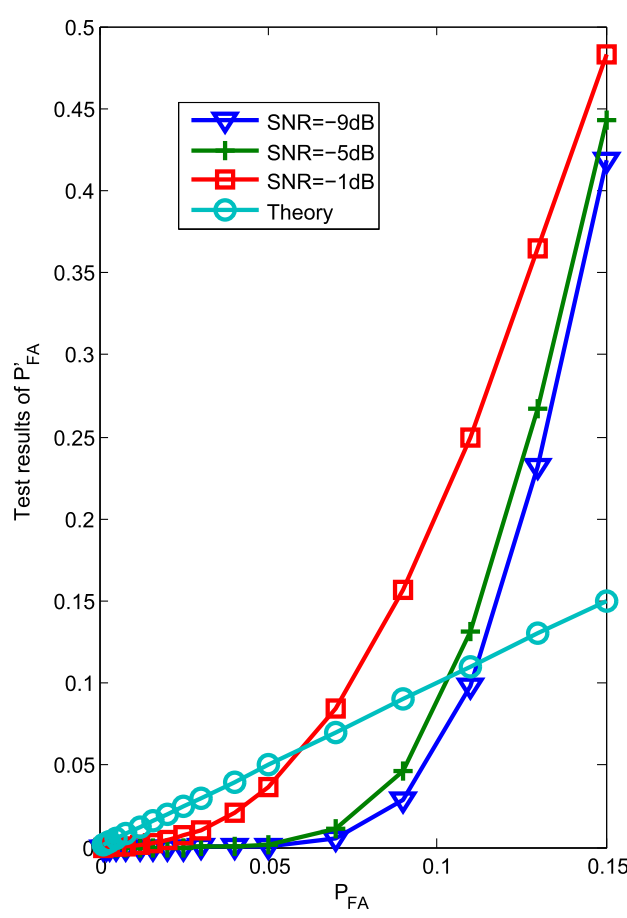

(a)

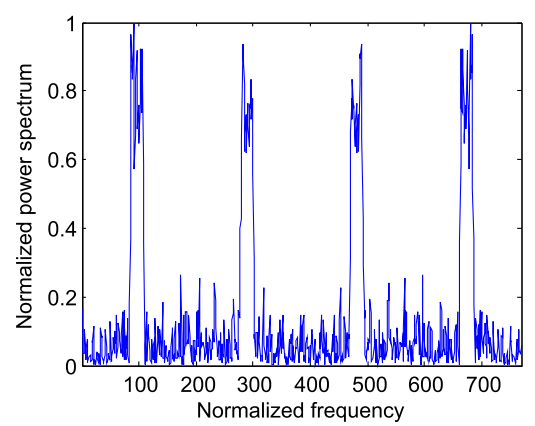

(b)

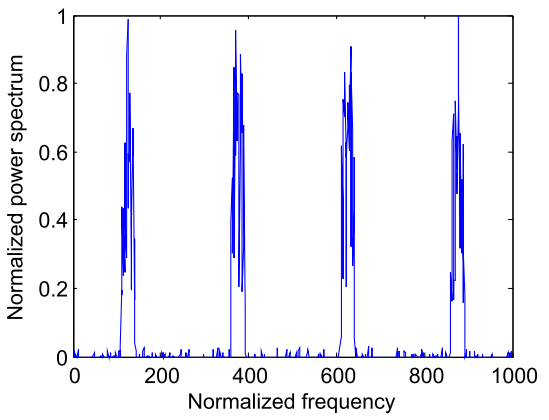

(c)

Figure 8 Spectrum sensing based on power spectrum estimation. (a) The test results of probability of false alarm for various SNR by the PSBS method. (b) Power spectrum estimation by PSBS, $M=100, N=250$. (c) Power spectrum estimation by CS-FAM, $M=400, N=1,000$. 


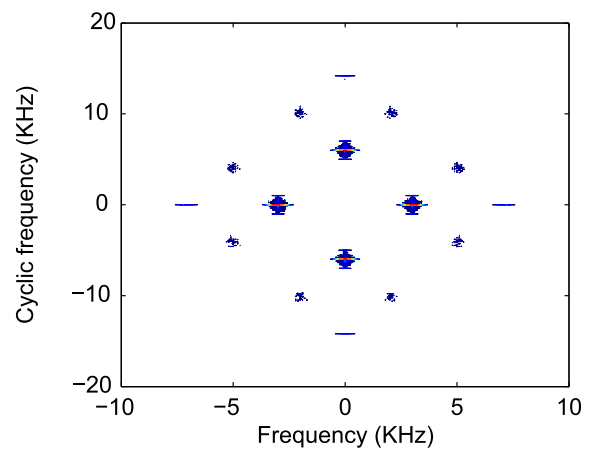

(a)

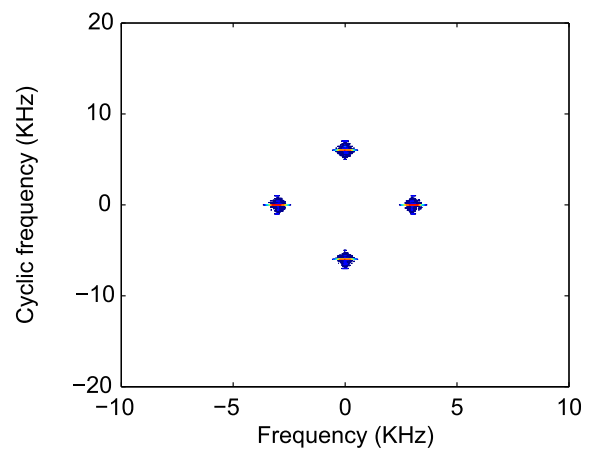

(c)

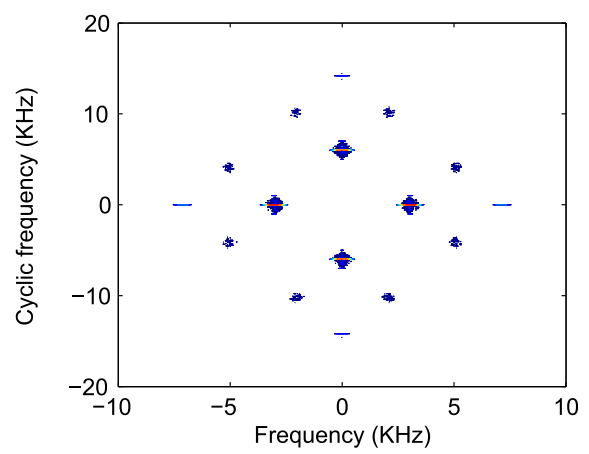

(b)

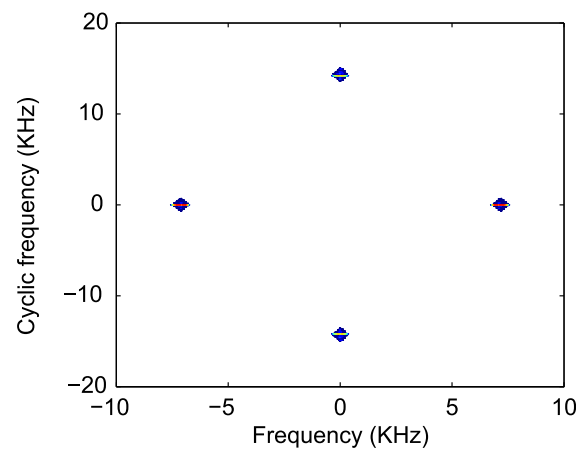

(d)

Figure 9 Cyclic feature detection by CS-FAM. (a) Cyclic spectrum estimation based on FAM from the Nyquist samples. (b) The whole cyclic spectrum estimation by CS-FAM without multiuser detection. (c-d) Two different BPSK signals are separated by CS-FAM with multiuser detection.

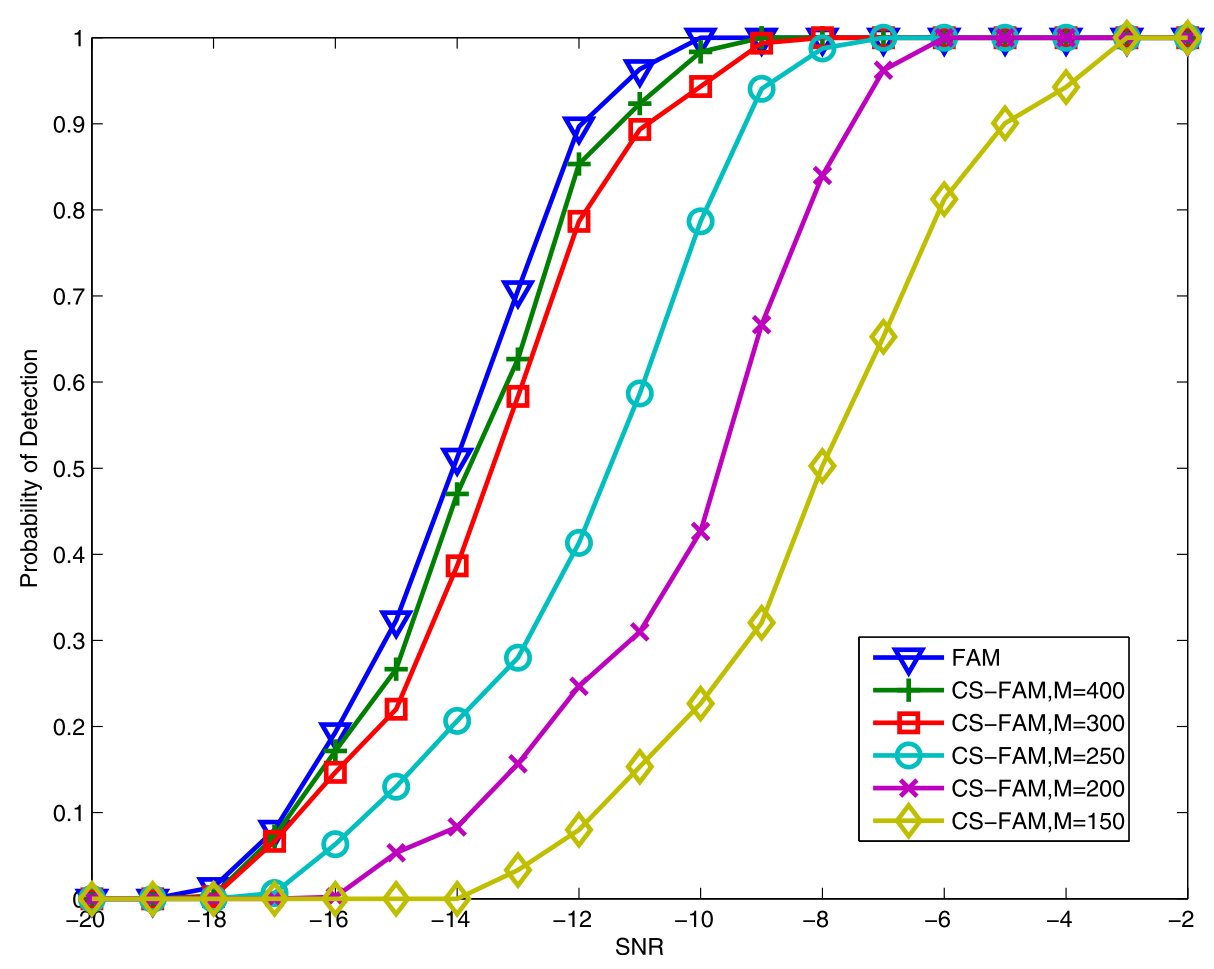

Figure 10 The BPSK identification performance of CS-FAM. 
in the inactive bands where there is no signal. This shortcoming is clearly shown in Figure 8b. Compared to the PSBS method, CS-FAM identifies a wide disparity between signals and noise, as shown in Figure 8c, which is advantageous for signal detection using a threshold. Another drawback of PSBS is that the size of the matrix used in this method is proportional to $3 M^{2} \times 3 N$, so the values of $M$ and $N$ chosen for PSBS were much smaller than those selected for CS-FAM, as shown in Figure 8. When using PSBS, a large $M$ value leads to high complexity, which further demonstrates that the PSBS method is not suitable for high spectral resolution sensing.

\subsection{Cyclic spectrum estimation performance}

Cyclic spectrum estimation is performed in the second step of CS-FAM, assuming that the active sub-bands have been detected in the first step. Here, we discuss the application of cyclic spectrum estimation from compressive samples. In the following tests, BPSK signals that exhibited spectral peaks at several $(f, \alpha)$ pairs in the cyclic spectrum were selected for analysis. The results showed that three distinct peaks are present at $\left(f_{c}, 1 / T_{b}\right),\left(0,2 f_{c}\right)$, and $\left(0,2 f_{c} \pm 1 / T_{b}\right)$. From these spectral peaks, the carrier frequency, symbol rate, and modulation format can be obtained.

The results of cyclic spectrum estimation using compressive samples are presented in Figure 8. In this test, the analog input contains two BPSK signals and the power of the weaker signal was one quarter that of the larger one. The carrier frequencies of the weaker and stronger signals were $f_{1}=3 \mathrm{kHz}$ and $f_{2}=7.1 \mathrm{kHz}$, respectively, where $N=660$ and $M=160$. Contour graphs of the estimation results are given in Figure 9. As Figure 9a shows, the cyclic spectrum of the two BPSK signals exhibits special peaks when using the FAM method with the Nyquist sampling. A similar estimation result is obtained using CS-FAM (from compressive samples without multiuser detection in the first step) is shown in Figure 9b. The number of the areas with a large magnitude was 16, including eight false areas yielded by the superposition of the two signals. Figure 9c, d presents the cyclic spectrum estimation results of each signal using CS-FAM with multiuser detection. The signal was correctly identified by the CS-FAM method, as shown in Figure 9c, d, whereas the FAM method failed to identify the peaks of the weaker signal, as shown in Figure 9a. Using FAM, a complex separating operation was required to identify the weaker signal correctly. In contrast, using the CS-FAM scheme, the smaller signal is separated during the first step and is not masked by the larger one.

In the next experiment, we examined the cyclic feature detection performance of CS-FAM using different compressive ratios. Three BPSK signals with different carrier frequencies were selected as analog inputs. A simple binary hypothesis test statistic was employed by

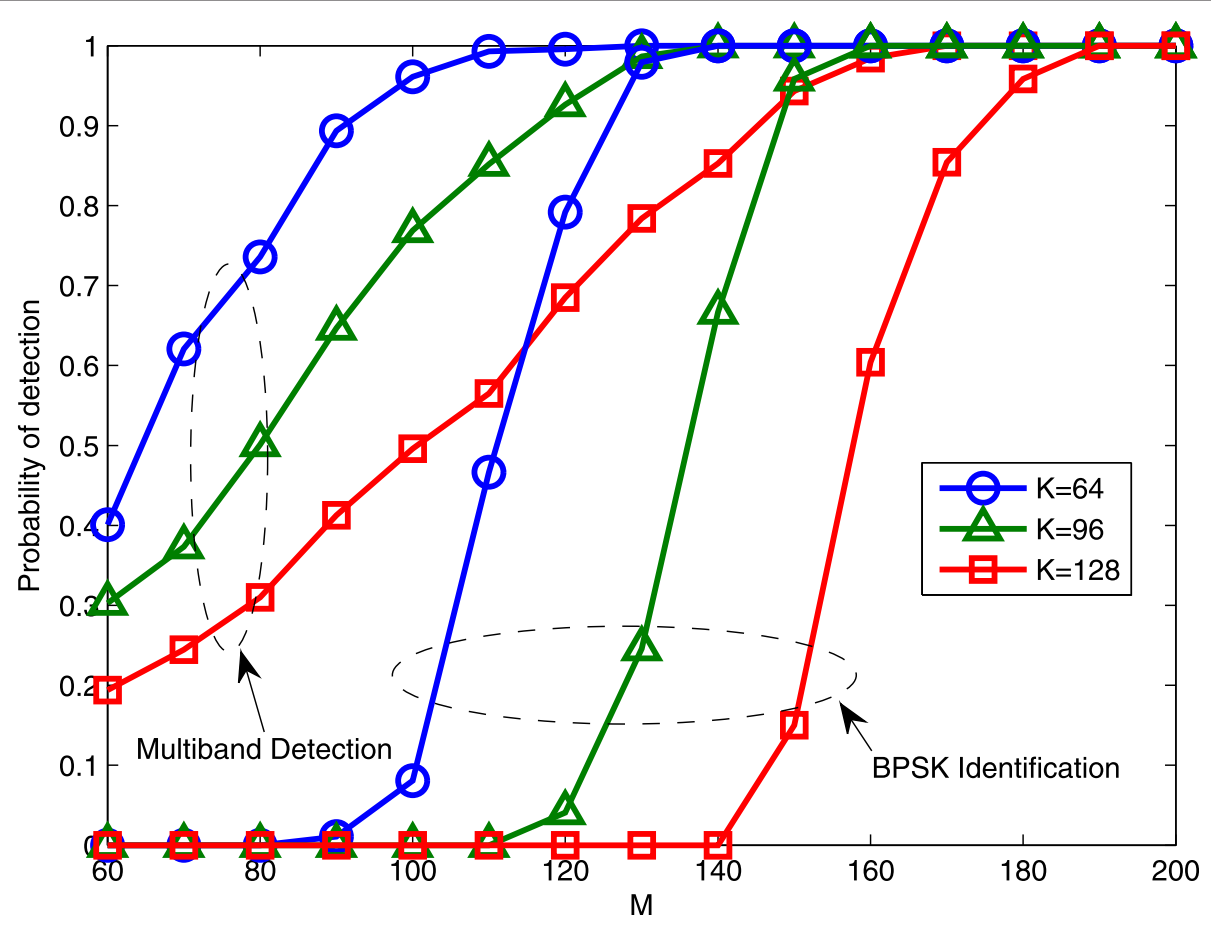

Figure 11 The performance of CS-FAM for various $\mathrm{K}$ and $\mathrm{M}$. 


$$
\begin{array}{lll}
H_{0}: & U^{\prime} \neq U \quad \text { format is not identified } \\
H_{1}: & U^{\prime}=U \quad \text { format is identified, }
\end{array}
$$

where $U$ is the reference set of a modulated signal from Table 2, i.e., $U=\left\{\left(f_{c}, 1 / T_{b}\right),\left(0,2 f_{c}\right),\left(0,2 f_{c} \pm 1 / T_{b}\right)\right\}$ if the input is a BPSK signal. $U^{\prime}$ is the set composed of the detected peaks. This matching decision method is stricter than the approaches used in [39] because the goal is to demonstrate cyclic spectrum estimation performance rather than signal classification. The resulting $U^{\prime}$ set contained four detected peaks for the BPSK signals. Figure 10 shows the symbol rates of the three BPSK signals, which were $5 f_{s} / N, N=500$, and $L=48$. To reconstruct the cyclic spectrum with an order similar to $N \times$ $N$, the methods in [20] require matrices proportional to $(500)^{4}$, which is unacceptable for common devices. It is clear that, when using CS-FAM, estimation quality improves with compressive ratio and that it converges towards the Nyquist rate obtained using FAM.

In Figure 11, a BPSK signal is selected with different bandwidth, indicated by $K$. SNR $=0 \mathrm{~dB}$ and $N=500$. The process of cyclic spectrum estimation requires more sampling branches than multiband detection does. Therefore, if the second step is operated effectively, the assumption of the active bands been detected is stated for (26). In addition, the results indicate that both of the steps require more sampling branches to maintain high performance when the wideband signal is less sparse.

\section{Conclusions}

This paper has introduced a joint scheme for low complexity cyclic spectrum estimation and robust multiband signal detection method based on compressive sampling. The process is divided into two steps. First, active sub-bands are identified from MMVs using joint sparse recovery and multiuser detection is performed using a bandwidth constraint. The proposed multiband detection method demonstrates striking robustness with sampling rate reduction and noise uncertainty. In the second step, the cyclic spectrums of each user are individually reconstructed. We also examine signal identification performance under different sampling rates and noise levels. The flexible nature of this scheme, accomplishing multiband and multiuser detection in its first step and parameter extractions in its second, allows for implementation in many practical applications.

\section{Competing interests}

The authors declare that they have no competing interests.

\section{Acknowledgements}

This work was supported by the Shanghai Provincial Natural Science Foundation of China under Grant No. 14ZR1447200, and the National Defense Foundation of China under Grant No. 9140C18010211Zxxx01 and No.9140C1801021xxx18. The authors also thank the anonymous reviewers and the editor for their valuable comments.
Received: 6 October 2014 Accepted: 17 November 2014

Published: 10 December 2014

\section{References}

1. L Neng-Jing, Z Yi-Ting, A survey of radar ECM and ECCM. IEEE Trans. Aero Electron Syst. 31(3), 1110-1120 (1995)

2. JA Lopez-Salcedo, JAD Peral-Rosado, G Seco-Granados, Survey on robust carrier tracking techniques. Commun. Surv. Tutor. IEEE 16(2), 670-688 (2014)

3. E Axell, G Leus, EG Larsson, HV Poor, Spectrum sensing for cognitive radio: stateof-the-art and recent advances. IEEE Signal Process. Mag. 29(3), 101-116 (2012)

4. H Sun, A Nallanathan, C-X Wang, Y Chen, Wideband spectrum sensing for cognitive radio networks: a survey. IEEE Wirel. Comm. Mag. 20(2), 74-81 (2013)

5. RG Baraniuk, Compressive sensing [lecture notes]. IEEE Signal Process. Mag. 24(4), 118-121 (2007)

6. S Foucart, H Rauhut, A Mathematical Introduction to Compressive Sensing (Springer, Boston, 2013)

7. M Mishali, YC Eldar, Blind multiband signal reconstruction: compressed sensing for analog signals. IEEE Trans. Signal Process. 57(3), 993-1009 (2009)

8. O Mehanna, ND Sidiropoulos, Frugal sensing: wideband power spectrum sensing from few bits. IEEE Trans. Signal Process. 61(10), 2693-2703 (2013)

9. G Leus, DD Ariananda, Power spectrum blind sampling. IEEE Signal Process Lett. 18(8), 443-446 (2011)

10. DD Ariananda, G Leus, Compressive wideband power spectrum estimation. IEEE Trans. Signal Process. 60(9), 4775-4789 (2012)

11. Y Miar, C D'Amours, T Aboulnasr, A novel reduced power compressive sensing technique for wideband cognitive radio. EURASIP J. Wirel. Commun. Netw. 2012(1), 281 (2012)

12. J Unnikrishnan, $W$ Veeravalli, Algorithms for dynamic spectrum access with learning for cognitive radio. IEEE Trans. Signal Process. 58(2), 750-760 (2010)

13. A Bourdoux, D Cabric, G Ferrari, F Horlin, Z Tian, Dynamic spectrum access: from the concept to the implementation. EURASIP J. Wirel. Commun. Netw. 2010(1), 654395 (2010)

14. D Noguet, L Biard, M Laugeois, Cyclostationarity detectors for cognitive radio: architectural tradeoffs. EURASIP J. Wirel. Commun. Netw. 2010(1), 526429 (2010)

15. OA Dobre, A Abdi, Y Bar-Ness, W Su, Survey of automatic modulation classification techniques: classical approaches and new trends. Commun. IET 1(2), 137-156 (2007)

16. Z Sun, R Liu, W Wang, Joint time-frequency domain cyclostationarity-based approach to blind estimation of OFDM transmission parameters. EURASIP J. Wirel. Commun. Netw. 2013(1), 117 (2013)

17. H Bolcskei, Blind estimation of symbol timing and carrier frequency offset in wireless OFDM systems. IEEE Trans. Commun. 49(6), 988-999 (2001)

18. D Cohen, E Rebeiz, V Jain, YC Eldar, D Cabric, Cyclostationary feature detection from sub-nyquist samples, in 4th IEEE International Workshop (2011) (IEEE, New York, USA, 2011), pp. 333-336

19. E Rebeiz, V Jain, D Cabric, Cyclostationary-based low complexity wideband spectrum sensing using compressive sampling, in Communications (ICC) (IEEE International Conference, IEEE, Ottawa, Canada, 2012), pp. 619-1623

20. Z Tian, Y Tafesse, BM Sadler, Cyclic feature detection with sub-Nyquist sampling for wideband spectrum sensing. IEEE J Sel Top Signal Process 6(1), 58-69 (2012)

21. L Safatly, B Aziz, A Nafkha, Y Louet, Y Nasser, A El-Hajj, K Kabalan, L Safatly, B Aziz, A Nafkha, Y Louet, Y Nasser, A El-Hajj, K Kabalan, Blind spectrum sensing using symmetry property of cyclic autocorrelation function: from theory to practice. EURASIP J. Wirel. Commun. Netw. 2014(1), 26 (2014)

22. RS Roberts, WA Brown, HH Loomis, Computationally efficient algorithms for cyclic spectral analysis. IEEE Signal Process. Mag. 8(2), 38-49 (1991)

23. AB MacKenzie, JH Reed, P Athanas, CW Bostian, RM Buehrer, LA DaSilva, SW Ellingson, YT Hou, M Hsiao, J-M Park, C Patterson, S Raman, C da Silva, Cognitive radio and networking research at Virginia Tech. Proc. IEEE 97(4), 660-688 (2009)

24. WA Gardner, Exploitation of spectral redundancy in cyclostationary signals. IEEE Signal Process. Mag. 8(2), 14-36 (1991)

25. WA Gardner, Statistical Spectral Analysis: A Nonprobabilistic Theory (Prentice-Hall, Englewood Cliffs, NJ, 1987)

26. RS Roberts, WA Brown Jr, HH Loomis, Cyclostationarity in Communications and Signal Processing (IEEE Press, Boston, 1994), pp. 455-479

27. VP Sathe, PP Vaidyanathan, Effects of multirate systems on the statistical properties of random signals. IEEE Trans. Signal Process. 41(1), 131-146 (1993) 
28. L Izzo, A Napolitano, Multirate processing of time series exhibiting higher order cyclostationarity. IEEE Trans. Signal Process. 46(2), 429-439 (1998)

29. A Sony, PP Vaidyanathan, Bifrequency and bispectrum maps: a new look at multirate systems with stochastic inputs. IEEE Trans. Signal Process. 48(3), 723-736 (2000)

30. S Kirolos, I Laska, M Wakin, M Duarte, D Baron, T Ragheb, Y Massoud, R Baraniuk, Analog-to-information conversion via random demodulation, in Design, Applications, Integration and Software, 2006 IEEE (CAS Workshop, Dallas, 2006), pp. 71-74

31. M Mishali, YC Eldar, Wideband spectrum sensing at sub-Nyquist rates [applications corner]. IEEE Signal Process. Mag. 28(4), 102-135 (2011)

32. SF Cotter, BD Rao, K Engan, K Kreutz-Delgado, Sparse solutions to linear inverse problems with multiple measurement vectors. IEEE Trans. Signal Process. 53(7), 2477-2488 (2005)

33. YC Eldar, M Mishali, Robust recovery of signals from a structured union of subspaces. IEEE Trans. Inform. Theor. 55(11), 5302-5316 (2009)

34. J Chen, X Huo, Theoretical results on sparse representations of multiplemeasurement vectors. IEEE Trans. Signal Process. 54(12), (2006)

35. JA Tropp, AC Gilbert, MJ Strauss, Algorithms for simultaneous sparse approximation. Part l: greedy pursuit. Signal Process. 86(3), 572-588 (2006)

36. H Urkowitz, Energy detection of unknown deterministic signals. Proc. IEEE 55(4), 523-531 (1967)

37. S Oymak, MA Khajehnejad, B Hassibi, Recovery threshold for optimal weight 11 minimization, in Information Theory Proceedings (ISIT), 2012 IEEE International Symposium (IEEE, Cambridge, USA), pp. 2032-2036

38. WA Gardner, W Brown, C-K Chen, Spectral correlation of modulated signals: part II-digital modulation. IEEE Trans. Comm. 35(6), 595-601 (1987)

39. B Ramkumar, Automatic modulation classification for cognitive radios using cyclic feature detection. IEEE Circ. Syst. Mag. 9(2), 27-45 (2009)

doi:10.1186/1687-1499-2014-218

Cite this article as: Pan et al:: Joint multiband signal detection and

cyclic spectrum estimation from compressive samples. EURASIP Journal on Wireless Communications and Networking 2014 2014:218.

\section{Submit your manuscript to a SpringerOpen ${ }^{\circ}$ journal and benefit from:}

- Convenient online submission

- Rigorous peer review

- Immediate publication on acceptance

- Open access: articles freely available online

- High visibility within the field

- Retaining the copyright to your article

Submit your next manuscript at $\gg$ springeropen.com 